\title{
Incidence and risk factors for chronic postsurgical pain following video-assisted thoracoscopic surgery: a retrospective study
}

Yingying Zhang ${ }^{\dagger}$, Rong Zhou ${ }^{\dagger}$, Bailing Hou, Suhong Tang, Jing Hao, Xiaoping Gu, Zhengliang Ma* and Juan Zhang*

\begin{abstract}
Background: Video-assisted thoracoscopic surgery (VATS) has been widely used as an alternative for thoracotomy, but the reported incidence of chronic postsurgical pain (CPSP) following VATS varied widely. The purpose of this study was to investigate the incidence and risk factors for CPSP after VATS.
\end{abstract}

Methods: We retrospectively collected preoperative demographic, anesthesiology, and surgical factors in a cohort of patients undergoing VATS between January 2018 and October 2020. Patients were interviewed via phone survey for pain intensity, and related medical treatment 3 months after VATS. Univariate and multivariate analysis were used to explore independent risk factors associated with CPSP.

Results: 2348 patients were included in our study. The incidence of CPSP after VATS were $43.99 \%$ ( $n=1033$ of 2348 ). Within those suffering CPSP, $14.71 \%$ ( $n=152$ of 1033) patients reported moderate or severe chronic pain. Only $15.23 \%$ $(n=23$ of 152) patients with moderate to severe chronic pain sought active analgesic therapies. Age $<65$ years (OR $1.278,95 \%$ Cl 1.057-1.546, $P=0.011$ ), female (OR 1.597, 95\% Cl 1.344-1.898, $P<0.001$ ), education level less than junior school (OR 1.295, 95\% Cl 1.090-1.538, $P=0.003)$, preoperative pain (OR 2.564, 95\% Cl 1.696-3.877, $P<0.001)$, consumption of rescue analgesia postoperative (OR 1.248, 95\% Cl 1.047-1.486, $P=0.013$ ), consumption of sedative hypnotic postoperative (OR 2.035, 95\% Cl 1.159-3.574, $P=0.013)$, and history of postoperative wound infection (OR 5.949, 95\% Cl 3.153-11.223, $P<0.001)$ were independent risk factors for CPSP development.

Conclusions: CPSP remains a challenge in clinic because half of patients may develop CPSP after VATS.

Trial registration Chinese Clinical Trial Registry (ChiCTR2100045765), 2021/04/24

Keywords: Chronic postsurgical pain, Video-assisted thoracoscopic surgery, Predictors, Multivariate logistic regression

\footnotetext{
*Correspondence: mazhengliang1964@nju.edu.cn; zhangjuannju@163.com †Yingying Zhang and Rong Zhou contributed equally to this work Department of Anesthesiology, Nanjing Drum Tower Hospital, The Affiliated Hospital of Nanjing University Medical School, 321 Zhongshan Road, Nanjing 210008, Jiangsu, China
}

\begin{abstract}
Introduction
Chronic postsurgical pain (CPSP) is defined as chronic pain that develops or increases in intensity after a surgical procedure or a tissue injury and persists beyond the healing process, i.e., at least 3 months after the surgery or tissue trauma by International Classification of Diseases-11 (ICD-11) [1], and its incidence varies from 3 to $85 \%$ according to surgery type [2]. As traumatic as thoracotomy, the reported incidence of CPSP can be
\end{abstract}


up to $57 \%$ [3]. Since video-assisted thoracoscopic surgery (VATS) was introduced into clinical practice in the early 1990s, it has been widely used as an alternative to thoracotomy over the past 30 years [4]. Though it was considered less injury than thoracotomy and with relief of postoperative acute pain, the incidence of CPSP after VATS has been reported to range from 7.7 to $50 \%$ [1]. Limited by small sample size and inconsistent followup time, the reported incidence of CPSP following VATS varies widely and we can't reach a comprehensive summary about the occurrence of CPSP after VATS at present.

The aetiology of CPSP after VATS is multifactorial and may involve both patient- and treatment-related factors. Although some studies have examined perioperative risk factors for the development of CPSP after VATS, most of them focused on a limited number of variables [5-7]. Since CPSP has been associated with long-term opioids use, unnecessary psychological pressure and reduced quality of life, identifying risk factors related to CPSP after VATS will be helpful for clinicians to carry out targeted prevention and help patients to form appropriate expectations [8].

Based on the above reasons, the primary aim of this study was to investigate the incidence of CPSP after VATS in a large sample of patients. The second aim was to identify independently predictors of CPSP from a comprehensive evaluation of demographic, anesthesiology, and surgical factors in a retrospective cohort.

\section{Methods}

\section{Study design and population}

This retrospective, observational study was approved by the Institutional Review Board (IRB) for Clinical Investigations at Nanjing Drum Tower Hospital, The Affiliated Hospital of Nanjing University Medical School (2020-297-02) and retrospective requirement for written informed consent was waived. This study was registered at Chinese Clinical Trial Registry (ChiCTR2100045765). Patients who underwent VATS between January 2018 and December 2020 at our institution (Nanjing Drum Tower Hospital, The Affiliated Hospital of Nanjing University Medical School) were identified. Study inclusion criteria were as follows: (1) age $\geq 18$ years; (2) American Society of Anesthesiologists(ASA) I-III grade; (3) non-emergency VATS surgery. The exclusion criteria were as follows: (1) patients with previous thoracodorsal surgery; (2) patients underwent bilateral surgery or converted to thoracotomy; (3) patients who developed III/ IV/V complications according to Clavien-Dindo grade system; (4) patients with inadequate medical records perioperatively.

\section{Data collection}

Data on demographics, medical history, anesthesia/ surgery related parameters, perioperative pain-related parameters were collected from an electronic medical records retrieval system.

Total intravenous anesthesia protocol was performed. No premedication was administered before surgery. Anesthesia was induced by intravenous midazolam $0.05 \mathrm{mg} /$ $\mathrm{kg}, 1 \%$ propofol $1-2 \mathrm{mg} / \mathrm{kg}$ (or etomidate $0.3-0.5 \mathrm{mg} / \mathrm{kg}$ ), fentanyl $3-8 \mu \mathrm{g} / \mathrm{kg}$, vecuronium bromide $0.08-0.12 \mathrm{mg} /$ $\mathrm{kg}$. Double-lumen endotracheal intubation was performed under visual laryngoscope. Anesthesia was maintained with continuous infusion of propofol $4-12 \mathrm{mg} / \mathrm{kg} / \mathrm{h}$, remifentanil $0.05-0.2 \mu \mathrm{g} / \mathrm{kg} / \mathrm{min}$, cisatracurium $1-3 \mu \mathrm{g} /$ $\mathrm{kg} / \mathrm{min}$. Intravenous patient-controlled analgesia consisted of a combination of sufentanil $1 \mu \mathrm{g} / \mathrm{mL}$, ondansetron $8 \mathrm{mg}$, and dexmedetomidine $10 \mathrm{mg}$ at a continuous infusion rate of $2 \mathrm{~mL} / \mathrm{h}$ and a bolus of $0.5 \mathrm{~mL}$ with a lockout interval of $15 \mathrm{~min}$. Based on the anesthesia record sheet, whether using nerve block, dexmedetomidine, and sevoflurane, dosage of fentanyl and remifentanil per kilogram, and intraoperative blood transfusion were collected.

VATS was performed by a two-port technique, one port was used as an operating hole and the other as an observation hole. The location of the chest wall incision was selected by surgeons according to the clinical features of patient's lesion. At the end of surgery, the chest tube was disposed for postoperative thoracic drainage. The etiology of the operation, duration of the operation, whether lymph node dissection was performed and the amount of intraoperative blood loss were collected according to the surgery records.

If there were no contraindications, all patients received intravenous injection of propacetamol hydrochloride after operation routinely. If patients complained severe pain, surgeons choosed indomethacin, flurbiprofen axetil injection, codeine phosphate, pethidine or tramadol as rescue analgesics according to the patient's pain severity. The use of rescue analgesics was extracted from the electronic medical system.

\section{Evaluation of CPSP}

Patients were contacted by telephone about whether CPSP developed after VATS. Our list of questions can be divided into two main parts:

Part 1. To confirm whether CPSP developed. The following questions were asked: 
1. Did you feel any pain around the surgery scar or the surrounding issue 3 months after VATS?

2. Did you feel pain before surgery? Was the pain 3 months after VATS the same as the preoperative pain?

3. Was there any other cause for the pain (recurrence of malignant tumor or chronic wound infection)?

Part 2. To evaluate the intensity of CPSP and record the patients' pain relief methods. The following questions were asked:

1. If 0 represents no pain and 10 represents the worst pain you can imagine, which number will reflects your most severe pain 3 months after VATS appropriately?

2. Did you adopt any measure to solve the pain, such as having a rest or reducing daily activity, taking medicine on yourself, asking help from doctors or none [9]?

If CPSP was confirmed by part 1 , further understanding of the patient's CPSP characteristics could be obtained through part 2. Numerical Rating Scale (NRS) was used to evaluate patients' pain intensity and the most severe pain could be induced when rest, coughing, moving or others. Pain score $\geq 1$ were diagnosed as CPSP [10].

\section{Statistical analysis}

Data were analyzed using SPSS 19.0 (SPSS, Inc., Chicago, IL, USA). The results were expressed as means \pm standard deviation for continuous variables or as number and percentages for categorical variables. The independent $t$ test or Mann-Whitney U-tests were used for between-group testing, depending on the distribution of the variables. Chi-square test tests or Fisher's exact tests were applied for categorical variables.

Univariable logistic regression analysis was applied to examine predictors of CPSP after VATS, and candidate covariates were chosen based on statistical significance or possible clinical importance. Multivariate model was developed using a stepwise forward approach. The discriminatory power of the multivariate model was evaluated by using the area under the receiver operating characteristic curve and its $95 \%$ confidence interval (CI). The calibration of the multivariate model was evaluated using the Hosmer-Lemeshow goodnessof-fit statistic, where a high $P$-value indicates good calibration. A significance level of $5 \%(P<0.05)$, and confidence intervals of $95 \%$ were used [11].

\section{Results}

Of 3147 patients who were screened for inclusion, 2738 met the inclusion criteria. When we attempted to contact these patients and assess their postoperative pain, 348 patients were excluded due to unanswered phone calls/refusal to answer, and 42 were excluded due to death. Finally, 2348 patients were included in our analysis (Fig. 1). According to the CPSP definition from ICD-11, $43.99 \%(n=1,033$ of 2348$)$ patients reported characteristics of CPSP after surgery with approximately $14.71 \%$ $(n=152$ of 1033) reported moderate to severe pain $(\mathrm{NRS}>3)$. Within those who reported moderate to severe pain, $22.52 \%(n=34$ of 152$)$ did not take any treatment, $62.25 \%(n=94$ of 152$)$ relieved their pain by resting or reducing activity, only $15.23 \%(n=23$ of 152$)$ sought active analgesic therapies (medicine or consultation to doctors).

Sociodemographic and medical history of patients were presented in Table 1. Factors associated with CPSP included age, sex, smoking history, drinking history, education level, and preoperative pain $(P<0.05)$.

Data about surgery and anesthesia were summarized in Table 2 . Statistically significant differences were observed, including the consumption of fentanyl dose per kilogram $(\mu \mathrm{g} / \mathrm{kg})$, blood loss volume intraoperative $(\mathrm{ml} /$ $\mathrm{kg}$ ), the consumption of rescue analgesia postoperative, the consumption of sedative hypnotic postoperative, and history of postoperative wound infection $(P<0.05)$.

Based on statistical significance or possible clinical implication, variates with $P<0.25$ in the univariate analysis were entered in the multivariate model. As showed in Fig. 2, seven risk factors were identified for CPSP after VATS: age $<65$ years (OR 1.278, 95\% CI 1.057-1.546, $P=0.011$ ), female (OR 1.597, 95\% CI 1.344-1.898, $\mathrm{P}<0.001$ ), education level less than junior school (OR $1.295,95 \%$ CI 1.090-1.538, $P=0.003$ ), preoperative pain (OR 2.564, 95\% CI 1.696-3.877, $P<0.001$ ), consumption of rescue analgesia postoperative (OR 1.248, 95\% CI 1.047-1.486, $P=0.013)$, consumption of sedative hypnotic postoperative (OR 2.035, 95\% CI 1.159-3.574, $P=0.013)$, and history of postoperative wound infection (OR 5.949, 95\% CI 3.153-11.223, $P<0.001$ ).

The predictive model for CPSP after VATS yield the area under the receiver operating characteristic curve of 0.622 (95\% CI 0.599-0.644) (Fig. 3), and the model showed good calibration by Hosmer-Lemeshow goodness-of-fit statistic with $X^{2}=4.956, \mathrm{P}=0.665$.

\section{Discussion}

Although VATS alleviates some iatrogenic injuries, it is still controversial whether this technique can reduce the risk of CPSP. The reported rates of CPSP after VATS 


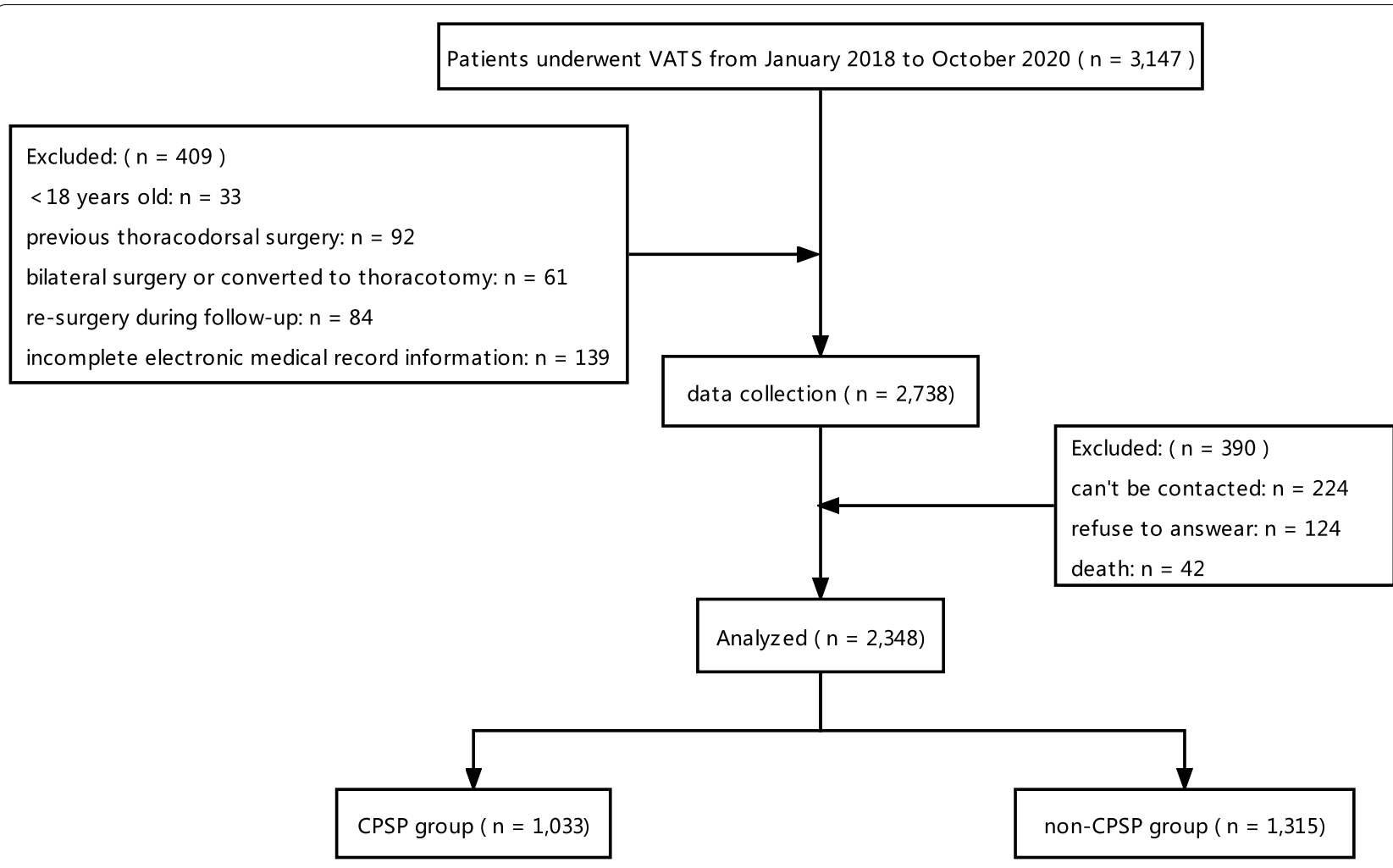

Fig. 1 Flow diagram for patient inclusion

varies widely, such a difference between studies may be related to the methodology (uni-portal vs. multi-portal VATS), perioperative analgesia regimen and CPSP definition. Additionally, "risk factor" has been a hot spot in the field of CPSP research in recent 10 years [12]. Surgical procedure, age, physical health, mental health, preoperative pain in the surgical field and another area have been proved to be risk factors for the occurrence of CPSP after several surgical procedures $[13,14]$.

In this study, we investigated the incidence of CPSP after two-ports VATS in 2348 patients. Our study showed that the incidence of CPSP was $43.99 \%$. Most CPSP were mild and bearable, and the incidence of moderate-severe CPSP were $14.71 \%$. The results of multivariate logistic regression analysis showed that age $<65$ years, female, education level less than junior school, preoperative pain, consumption of rescue analgesia postoperative, consumption of sedative hypnotic postoperative, and history of postoperative wound infection were risk factors of CPSP.

Previously published meta-analysis reported the incidence of CPSP after thoracotomy was $57 \%$, but data about the risk of developing CPSP after VATS were not sufficient to summarize $[3,15]$. The reported incidences of CPSP after VATS range from 7.7 to $50 \%$ [1], apparently, our result ranks at a higher level of it. The relatively higher incidence may be explained by the definition of CPSP we used. The postoperative CPSP we calculated included pain of any intensity. Although some researchers believe that pain $>3$ points is clinically significant, study has confirmed that CPSP of any intensity will lead to a decline in postoperative quality of life [8]. From our perspective, taking into account pain of 1-2 points will prevent clinicians and patients from ignoring those bothering mild CPSP. In addition to the high prevalence of CPSP, we were surprised to find that the majority of patients deal with CPSP in a negative way, only $15.23 \%$ of patients with moderate to severe pain sought active analgesic therapies (medicine or consultation to doctors), most of the remaining patients got relief from CPSP by resting or reducing activity. Overall, our results showed that although VATS significantly reduced the iatrogenic injury caused by surgical procedures, the incidence of CPSP after VATS remained high and most patients had a negative attitude towards CPSP management.

In terms of demographics data, age $<65$ years, female and education level less than junior school were independent risk factors for CPSP. These results were consistent with previously published, known risk factors for CPSP in a variety of surgical procedures, including 
Table 1 Sociodemographic and medical history of subjects without and with CPSP $(n=2348)$

\begin{tabular}{|c|c|c|c|}
\hline Variables & $\begin{array}{l}\text { Non-CPSP } \\
(n=1315)\end{array}$ & $\operatorname{CPSP}(n=1033)$ & $P$ value \\
\hline \multicolumn{4}{|l|}{ Age, n (\%) } \\
\hline$<65$ & $897(68.2)$ & $757(73.3)$ & \multirow[t]{2}{*}{0.008} \\
\hline$\geq 65$ & $418(31.8)$ & $276(26.7)$ & \\
\hline Female, n (\%) & $627(47.7)$ & $623(60.3)$ & $<0.001$ \\
\hline \multicolumn{4}{|l|}{ BMI, n (\%) } \\
\hline$<24 \mathrm{~kg} / \mathrm{m}^{2}$ & $722(54.9)$ & $605(58.6)$ & \multirow[t]{2}{*}{0.076} \\
\hline$\geq 24 \mathrm{~kg} / \mathrm{m}^{2}$ & $593(45.1)$ & $428(41.4)$ & \\
\hline \multicolumn{4}{|l|}{ ASA, n (\%) } \\
\hline$|/| \mid$ & $156(11.9)$ & $98(9.5)$ & \multirow[t]{2}{*}{0.118} \\
\hline III & $1159(88.1)$ & $931(90.5)$ & \\
\hline Smoking history, n (\%) & $200(15.2)$ & $121(11.7)$ & 0.014 \\
\hline Drinking history, n (\%) & $143(10.9)$ & $84(8.1)$ & 0.026 \\
\hline Hypertension, n (\%) & $358(27.2)$ & $273(26.4)$ & 0.666 \\
\hline Diabetes mellitus, n (\%) & $128(9.7)$ & $92(8.9)$ & 0.494 \\
\hline CHD, n (\%) & $36(2.7)$ & $20(1.9)$ & 0.206 \\
\hline Surgery history, n (\%) & $413(31.4)$ & $413(31.2)$ & 0.903 \\
\hline Preoperative pain, n (\%) & $36(2.7)$ & $75(7.3)$ & $<0.001$ \\
\hline \multicolumn{4}{|c|}{ Education level less than junior school, n (\%) } \\
\hline & $658(50.0)$ & $591(57.2)$ & 0.001 \\
\hline \multicolumn{4}{|c|}{ Consumption of sedative hypnotic preoperative, n (\%) } \\
\hline & $458(34.8)$ & $338(32.7)$ & 0.284 \\
\hline
\end{tabular}

bold: $P<0.05$

$B M I$ body mass index, ASA American Society of Anesthesiologists, $C H D$ coronary heart disease

thoracic surgery $[13,14,16]$.The relationship between age and CPSP can be explained by two points. First, young patients are biologically more sensitive to low-intensity noxious stimuli and may having a more heightened central nervous system responsiveness [17]. Second, from a physiological perspective, older adults are more conservative in pain perception and reporting and are more reluctant to report pain when it dose occurs [18]. The association between sex and CPSP can also be explained from these two aspects. From biologically, differences of sex hormone levels, pain-related receptor activity such as $N$-methyl- $D$-aspartic acid receptor or $P 2 X 3$ receptor, $\mu / \kappa$ subtype splits in the endogenous analgesic system, and brain structure and function between men and women are related to the mechanism of sex differences in pain perception [8]. And from psychologically, female are more self-conscious and more likely to report pain to others [19]. Association between education level and CPSP has also been proved in several studies, but the specific mechanism is still unclear, pain catastrophizing may be a mediating factor between them [20]. Anyway, the combination of these demographic factors suggests a higher risk of developing CPSP. Recognition of these predictors, although unmodifiable, can help clinicians identify high-risk groups during preoperative evaluation and tailor an individualized pain treatment regimen [21].

As for perioperative pain-related parameters, our findings revealed that preoperative pain, the consumption of rescue analgesics and sedative hypnotic after surgery were independent predictors for CPSP. The association between preoperative pain and CPSP has been reported in several clinical trials $[3,13,22]$. The mechanism between preoperative pain and CPSP remains elusive but cumulative evidence has demonstrated that sensitization of the peripheral and central nervous system, which related to the alterations of peripheral nociceptors sensitivity and function of the pain descending inhibitory system may be the possible explanations between them [23]. Since preoperative pain could explain part of the interindividual variance in pain sensitivity, it may sensitize patients to new painful stimuli [24].

Postoperative use of rescue analgesics is an important indicator of postoperative pain intensity, especially when pain scores are performed only once or twice a day, the need for rescue analgesics helps in revealing the true level of postoperative pain intensity [25]. Consistent with the thoracic surgery and other postsurgical chronic pain conditions, we reported that postoperative consumption of rescue analgesics and sedative hypnotic in hospital (which indicates a higher severity of acute pain) was associated with a greater risk of developing CPSP [26]. Acute postoperative pain represents actual or potential tissue injury and motivates a response that removes the organism from such noxious stimuli [27]. The more severe the postoperative acute pain, the more severe the tissue injury, and the less adequate the pain control, which may induce peripheral sensitization and neuroplastic changes that involves altered pain processing [28]. An interesting study reported that although acute pain after thoracic surgery was comprised of thoracic pain, shoulder pain, and referred pain, only thoracic pain was closely related to the occurrence of CPSP. This study further highlights the importance of effective management of postoperative acute pain [29]. Although our results did not show that nerve block reduces the risk of CPSP, the latest metaanalysis did report a meaningful change in the incidence of CPSP by controlling postoperative acute pain through regional anesthesia [30]. Discrepancy between results may be related to differences in nerve block technique, and the type of study design. Anyway, the attempts to better manage postoperative acute pain are of great clinical significance in CPSP prevention.

For surgery related parameters, the history of postoperative wound infection was strongly associated with the occurrence of CPSP. On the one hand, nociceptor neurons themselves can detect pathogens and their 
Table 2 Surgery and anesthesia data of subjects without and with CPSP $(n=2348)$

\begin{tabular}{|c|c|c|c|}
\hline Variables & Non-CPSP $(n=1315)$ & $\operatorname{CPSP}(n=1033)$ & $P$ value \\
\hline \multicolumn{4}{|l|}{ Anesthesia, n (\%) } \\
\hline General anesthesia & $965(73.4)$ & $771(74.6)$ & \multirow[t]{2}{*}{0.492} \\
\hline Combined with nerve block & $350(26.6)$ & $262(25.4)$ & \\
\hline Fentanyl dosage ( $\mu \mathrm{g} / \mathrm{kg})$ & $11.3 \pm 4.8$ & $11.0 \pm 4.3$ & 0.044 \\
\hline Remifentanil dosage ( $\mu \mathrm{g} / \mathrm{kg})$ & $11.8 \pm 10.1$ & $12.1 \pm 9.3$ & 0.478 \\
\hline Dexmedetomidine usage, $\mathrm{n}(\%)$ & $1214(92.3)$ & $960(92.9)$ & 0.573 \\
\hline Sevoflurane usage, n (\%) & $221(16.8)$ & $199(19.3)$ & 0.123 \\
\hline PCIA, n (\%) & $955(72.6)$ & $763(73.9)$ & 0.501 \\
\hline \multicolumn{4}{|l|}{ Surgical Procedure, n (\%) } \\
\hline Lung & 1235 (93.9) & $967(93.6)$ & \multirow[t]{3}{*}{0.179} \\
\hline Mediastinal & $76(5.8)$ & $57(5.5)$ & \\
\hline Others & $4(0.3)$ & $9(0.9)$ & \\
\hline Lymph node dissection, n (\%) & $603(56.2)$ & $470(43.8)$ & 0.863 \\
\hline Duration of surgery (min) & $106.4 \pm 47.6$ & $107.9 \pm 44.9$ & 0.189 \\
\hline Blood loss (ml/kg) & $2.0 \pm 4.1$ & $1.8 \pm 3.5$ & 0.013 \\
\hline Infusion volume (ml/kg) & $22.7 \pm 8.8$ & $22.1 \pm 8.0$ & 0.114 \\
\hline \multicolumn{4}{|c|}{ Consumption of rescue analgesia postoperative, $n(\%)$} \\
\hline & $450(34.2)$ & $411(39.8)$ & 0.005 \\
\hline \multicolumn{4}{|c|}{ Consumption of sedative hypnotic postoperative, n (\%) } \\
\hline & $22(1.7)$ & $32(3.1)$ & 0.022 \\
\hline \multicolumn{4}{|c|}{ Subcutaneous emphysema postoperative, $n(\%)$} \\
\hline & $210(16.0)$ & $185(17.9)$ & 0.212 \\
\hline \multicolumn{4}{|c|}{ History of postoperative wound infection, n (\%) } \\
\hline & $12(0.9)$ & $57(5.5)$ & $<0.001$ \\
\hline \multicolumn{4}{|c|}{ Postoperative pulmonary infection, $\mathrm{n}(\%)$} \\
\hline & $31(2.4)$ & $37(3.6)$ & 0.079 \\
\hline Postoperative WBC $\left(10^{9}\right)$ & $11.4 \pm 3.1$ & $11.3 \pm 3.3$ & 0.202 \\
\hline Postoperative CRP (mg/L) & $52.9 \pm 1.0$ & $51.1 \pm 1.1$ & 0.205 \\
\hline PONV, n (\%) & $180(13.7)$ & $149(14.4)$ & 0.610 \\
\hline
\end{tabular}

bold: $P<0.05$

$P C I A$ postoperative patient-controlled intravenous analgesia, WBC white blood cell, CRP C-reactive protein, $P O N V$ postoperative nausea and vomiting

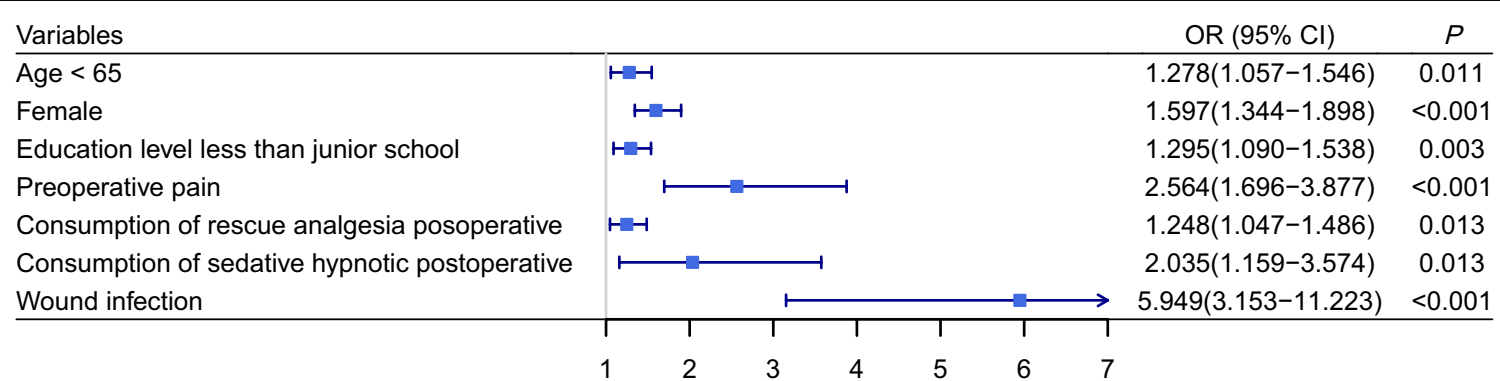

Fig. 2 Multivariate model for CPSP after VATS

related molecular ligands to mediate pain, on the other hand, immune cells secrete multiple cytokines during infection which can lower the threshold of action potentials of nociceptor [31]. In addition, cytokines and chemokines produced by activation of spinal astrocytes and microglias have been shown to be associated with chronic pain after infection, although the model used in this study was parasitic infection [32]. Peripheral and central sensitization due to infection may be responsible for the higher risk of CPSP. Our results showed no 


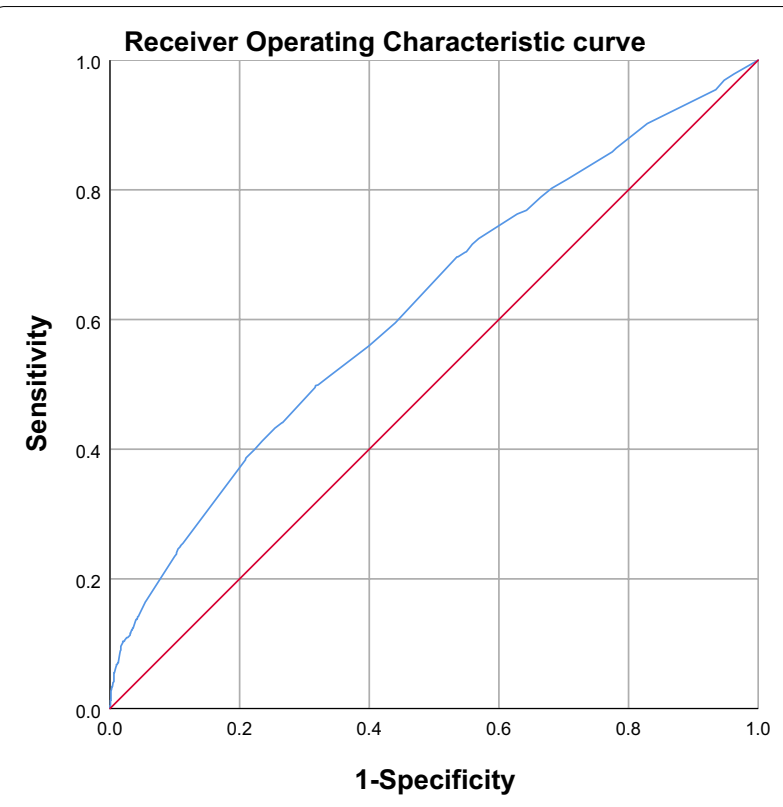

Fig. 3 The area under the ROC curve of CPSP multivariate model

statistically significant difference in the contents of white blood cells, lymphocytes, neutrophils and CRP between the CPSP group and the non-CPSP group after surgery as Wang et al. [33], but this may be related to the fact that we only routinely rechecked relevant biochemical indicators on the first day postoperative. After discharge, some patients would suffer from out-of-hospital wound infection due to improper nursing or pleural effusion. However, due to the defects of retrospective study, we could not obtain relevant data. Although current studies have found a strong association between infection and acute pain, the specific mechanisms between infection and chronic pain need a further investigation.

Guidelines recommend intravenous use of dexmedetomidine during VATS and a number of studies have found that intraoperative use of dexmedetomidine can reduce pain within $24 \mathrm{~h}$ after VATS without significant adverse events [34], however the relationship between intraoperative use of dexmedetomidine and CPSP after VATS has not been specifically discussed. Although we didn't find positive association between continuous pumping of dexmedetomidine with improvement in postoperative CPSP, it is worth noting that, as a routine medication, dexmedetomidine was used in about $92 \%$ of patients in our study population, which may have an impact on our results. A randomized controlled trial is needed to explore whether dexmedetomidine can lower the risk of CPSP after VATS.
Our study has strengths, including the large sample size and adequate range of risk factors collection. However, the results should still be interpreted cautiously for some reasons, mostly related to its retrospective design and the telephone interview. To solve these, We made a data acquisition table in advance to collect comprehensive and standardized data before, during and after surgery for each patient. During the telephone interview, we assessed patients' CPSP according to a pre-formulated list of questions, which ensured the homogeneity of the follow-up process. Besides, due to the long follow-up time of CPSP, other authors often use telephone follow-up to obtain relevant data $[8,35]$. Additionally, for the data collected from in a single institution, the results may be influenced by the selection bias.

\section{Conclusion}

Overall, our study found that the incidence of CPSP after VATS was $43.99 \%$. Although the majority of patients reported mild pain, such a high incidence suggests that CPSP after VATS remains an important challenge that cannot be ignored. In addition, our study found that younger age, female, low education level, preoperative pain, postoperative consumption of rescue analgesics and sedative hypnotic and postoperative wound infection were important predictors of CPSP, which suggested that clinicians should conducted a throughout evaluation perioperatively in order to identify high-risk groups of CPSP as soon as possible and tailor individualized pain prevention and treatment strategies for them.

\section{Abbreviations}

VATS: Video-assisted thoracoscopic surgery; CPSP: Chronic postsurgical pain Cl: Confidence interval; OR: Odds ratio; ICD-11: International Classification of Diseases-11; IRB: The Institutional Review Board; ASA: American Society of Anesthesiologists; NRS: Numerical Rating Scale; BMI: Body mass index; CHD: Coronary heart disease; PCIA: Postoperative patient-controlled intravenous analgesia; WBC: White blood cell; CRP: C-reactive protein; PONV: Postoperative nausea and vomiting.

\section{Acknowledgements \\ Not applicable.}

\section{Authors' contributions}

BLH and ZLM contributed to the study conception and design. Material preparation, data collection and analysis were performed by $Y Y Z, R Z$, SHT and $J H$. The first draft of the manuscript was written by $Y Y Z$ and JZ. All authors read and approved the final manuscript.

\section{Funding}

The study and the journal's Rapid Service Fee were supported by the National Natural Science Foundation of China (Grant No. 81471129, 81971044).

Availability of data and materials

The datasets generated during and/or analyzed during the current study are available from the corresponding author on reasonable request. 


\section{Declarations}

\section{Ethics approval and consent to participate}

The study was conducted according to the Helsinki Declaration and approved by the ethics community of Institutional Review Board (IRB) for Clinical Investigations at Nanjing Drum Tower Hospital, The Affiliated Hospital of Nanjing University Medical School (2020-297-02) and retrospective requirement for written informed consent was waived.

\section{Consent for publication}

Not applicable.

\section{Competing interests}

The authors declare no competing interests.

Received: 17 September 2021 Accepted: 18 February 2022

Published online: 02 March 2022

\section{References}

1. Tong $Y$, Wei P, Wang S, Sun Q, Cui Y, Ning N, Chen S, He X. Characteristics of postoperative pain after VATS and pain-related factors: the experience in National Cancer Center of China. J Pain Res. 2020;13:1861-7.

2. Yoon S, Hong WP, Joo H, Kim H, Park S, Bahk JH, Lee HJ. Long-term incidence of chronic postsurgical pain after thoracic surgery for lung cancer: a 10-year single-center retrospective study. Reg Anesth Pain Med. 2020;45(5):331-6.

3. Bayman EO, Parekh KR, Keech J, Selte A, Brennan TJ. A prospective study of chronic pain after thoracic surgery. Anesthesiology. 2017;126(5):938-51.

4. Handy JR Jr, Asaph JW, Douville EC, Ott GY, Grunkemeier GL, Wu Y. Does video-assisted thoracoscopic lobectomy for lung cancer provide improved functional outcomes compared with open lobectomy? Eur J Cardio-thoracic Surg. 2010;37(2):451-5.

5. Hirai $\mathrm{K}$, Usuda J. Uniportal video-assisted thoracic surgery reduced the occurrence of post-thoracotomy pain syndrome after lobectomy for lung cancer. J Thorac Dis. 2019;11(9):3896-902.

6. Wildgaard K, Ringsted TK, Hansen HJ, Petersen RH, Kehlet H. Persistent postsurgical pain after video-assisted thoracic surgery-an observational study. Acta Anaesthesiol Scand. 2016:60(5):650-8.

7. Bayman EO, Parekh KR, Keech J, Larson N, Vander Weg M, Brennan TJ. Preoperative patient expectations of postoperative pain are associated with moderate to severe acute pain after VATS. Pain Med (Malden, Mass). 2019;20(3):543-54

8. Fiorelli S, Cioffi L, Menna C, Ibrahim M, De Blasi RA, Rendina EA, et al. Chronic pain after lung resection: risk factors, neuropathic pain, and quality of life. J Pain Symptom Manage. 2020;60(2):326-35.

9. Kumar SP. Utilization of brief pain inventory as an assessment tool for pain in patients with cancer: a focused review. Indian J Palliat Care. 2011;17(2):108-15

10. Bendixen M, Jørgensen OD, Kronborg C, Andersen C, Licht PB. Postoperative pain and quality of life after lobectomy via video-assisted thoracoscopic surgery or anterolateral thoracotomy for early stage lung cancer: a randomised controlled trial. Lancet Oncol. 2016;17(6):836-44.

11. Rice DA, Kluger MT, McNair PJ, Lewis GN, Somogyi AA, Borotkanics R, et al. Persistent postoperative pain after total knee arthroplasty: a prospective cohort study of potential risk factors. Br J Anaesth. 2018;121(4):804-12.

12. Li Q, Dai W, Chen X, Su D, Yu W, Gu X. Publication trends and hot spots in chronic postsurgical pain (CPSP) research: a 10-year bibliometric analysis[J]. J Pain Res. 2021;14:2239-47.

13. Montes A, Roca G, Cantillo J, Sabate S. Presurgical risk model for chronic postsurgical pain based on 6 clinical predictors: a prospective external validation. Pain. 2020;161(11):2611-8.

14. Montes A, Roca G, Sabate S, Lao JI, Navarro A, Cantillo J, et al. Genetic and clinical factors associated with chronic postsurgical pain after hernia repair, hysterectomy, and thoracotomy: a two-year multicenter cohort study. Anesthesiology. 2015;122(5):1123-41.

15. Li X, Liu Y, Zhao J, Xiang Z, Ren C, Qiao K. The safety and efficacy of ultrasound-guided serratus anterior plane block (SAPB) combined with dexmedetomidine for patients undergoing video-assisted thoracic surgery (VATS): a Randomized controlled trial. J Pain Res. 2020;13:1785-95.
16. Gärtner R, Jensen M Nielsen J Ewertz M, Kroman N Kehlet H Prevalence of and factors associated with persistent pain following breast cancer surgery. JAMA. 2009:302(18):1985-92.

17. Bruce J, Thornton AJ, Powell R, Johnston M, Wells M, Heys SD, et al. Psychological, surgical, and sociodemographic predictors of pain outcomes after breast cancer surgery: a population-based cohort study. Pain. 2014:155(2):232-43

18. Yong HH, Gibson SJ, Horne DJ, Helme RD. Development of a pain attitudes questionnaire to assess stoicism and cautiousness for possible age differences. J Gerontol B Psychol Sci Soc Sci. 2001;56(5):P279-284.

19. Wandner LD, Scipio CD, Hirsh AT, Torres CA, Robinson ME. The perception of pain in others: how gender, race, and age influence pain expectations. $J$ Pain. 2012;13(3):220-7.

20. Aily JB, de Almeida AC, Ramírez PC, da Silva AT, Mattiello SM. Lower education is an associated factor with the combination of pain catastrophizing and kinesiophobia in patients with knee osteoarthritis? Clin Rheumatol. 2021;40(6):2361-7.

21. Gatchel RJ, McGeary DD, McGeary CA, Lippe B. Interdisciplinary chronic pain management: past, present, and future. Am Psychol. 2014;69(2):119-30.

22. Mathes T, Pape-Köhler C, Moerders L, Lux E, Neugebauer EAM. External validation and update of the RICP-A multivariate model to predict chronic postoperative pain. Pain Med (Malden, Mass). 2018;19(8):1674-82.

23. Kim DH, Pearson-Chauhan KM, McCarthy RJ, Buvanendran A. Predictive factors for developing chronic pain after total knee arthroplasty. J Arthroplasty. 2018:33(11):3372-8

24. Kaunisto MA, Jokela R, Tallgren M, Kambur O, Tikkanen E, Tasmuth T, et al. Pain in 1,000 women treated for breast cancer: a prospective study of pain sensitivity and postoperative pain. Anesthesiology. 2013;119(6):1410-21.

25. Steinthorsdottir KJ, Wildgaard L, Hansen HJ, Petersen RH, Wildgaard K. Regional analgesia for video-assisted thoracic surgery: a systematic review. Eur J Cardio-thoracic Surg. 2014:45(6):959-66.

26. Glare P, Aubrey KR, Myles PS. Transition from acute to chronic pain after surgery. Lancet (London, England). 2019;393(10180):1537-46.

27. Jin J, Peng L, Chen $Q$, Zhang D, Ren L, Qin P, et al. Prevalence and risk factors for chronic pain following cesarean section: a prospective study. BMC Anesthesiol. 2016;16(1):99.

28. Liu Y, Zhou M, Zhu X, Gu X, Ma Z, Zhang W. Risk and protective factors for chronic pain following inguinal hernia repair: a retrospective study. J Anesth. 2020;34(3):330-7.

29. Blichfeldt-Eckhardt MR, Andersen C, Ørding H, Licht PB, Toft P. From acute to chronic pain after thoracic surgery: the significance of different components of the acute pain response. J Pain Res. 2018:11:1541-8.

30. Levene JL, Weinstein EJ, Cohen MS, Andreae DA, Chao JY, Johnson M, et al. Local anesthetics and regional anesthesia versus conventional analgesia for preventing persistent postoperative pain in adults and children: a Cochrane systematic review and meta-analysis update. J Clin Anesth. 2019:55:116-27.

31. Chiu IM. Infection, pain, and itch. Neurosci Bull. 2018;34(1):109-19.

32. Borghi SM, Fattori V, Pinho-Ribeiro FA, Domiciano TP, Miranda-Sapla MM, Zaninelli $\mathrm{TH}$, et al. Contribution of spinal cord glial cells to L. amazonensis experimental infection-induced pain in BALB/c mice. J Neuroinflammation. 2019;16(1):113.

33. Wang HT, Liu W, Luo AL, Ma C, Huang YG. Prevalence and risk factors of chronic post-thoracotomy pain in Chinese patients from Peking Union Medical College Hospital. Chin Med J (Engl). 2012;125(17):3033-8.

34. Feray S, Lubach J, Joshi GP, Bonnet F, Van de Velde M. PROSPECT Working Group *of the European Society of Regional Anaesthesia and Pain Therapy. PROSPECT guidelines for video-assisted thoracoscopic surgery: a systematic review and procedure-specific postoperative pain management recommendations. Anaesthesia. 2022;77(3):311-25.

35. Cattoni M, Rotolo N, Mastromarino MG, Cardillo G, Nosotti M, Mendogni P, et al. Chronic chest pain and paresthesia after video-assisted thoracoscopy for primary pneumothorax. JThorac Dis. 2021;13(2):613-20.

\section{Publisher's Note}

Springer Nature remains neutral with regard to jurisdictional claims in published maps and institutional affiliations. 